\title{
El estudio científico del estrés crónico en neurociencias y psicooncología ${ }^{1}$
}

\author{
Manolete Moscoso \\ University of South Florida
}

Recibido: 30 de mayo de 2014 / Aprobado: 15 de junio de 2014

Presentamos un análisis de las limitaciones metodológicas del estudio del estrés $y$ del concepto de homeostasis. También, se describe un nuevo modelo acerca del estrés crónico, allostasis y carga allostática, que explica las interacciones entre el cerebro y los sistemas nervioso, endocrino e inmune en las causas de enfermedades físicas y mentales. Ilustramos empíricamente, con base en nuestras investigaciones, cómo este nuevo marco metodológico de investigación científica permite la integración multidisciplinaria de las ciencias biomédicas y las ciencias del comportamiento, reflejando el gran avance científico actual observado en el campo de las neurociencias y de la psicooncología. Se examina el impacto negativo del estrés crónico en la salud del individuo, poniendo énfasis en el rol central que cumple el concepto de distrés emocional percibido. Presentamos la terapia cognitiva centrada en mindfulness como una respuesta de tratamiento multidisciplinario en las áreas de la neurociencia y la psicooncología.

estrés crónico / allostasis / mindfulness / neurociencia / psico-oncología

\section{The scientific study of chronic stress in neuroscience and psycho-oncology}

The theory of allostasis and allostasis load allows a new integrative and multidisciplinary framework of the chronic stress process that identifies the brain as the mediator of the interactions among the central nervous system, the endocrine and immune systems in the development of disease. We examined the negative impact of chronic stress and the perceived emotional distress on illness. We made comments on our multidisciplinary research on mindfulness, as an illustration of these neurobiological and psychosocial interactions; and how this therapeutic approach facilitates the integration of the biomedical and behavioral sciences, as well as the integration of our current research findings in neuroscience and psychooncology.

chronic stress / allostasis / mindfulness / neuroscience / psycho-oncology

\footnotetext{
1 El presente estudio ha sido financiado por la Fundación Susan G. Komen for Breast Cancer Grant \# BCTRO201400.

Correo electrónico: mmoscoso@health.usf.edu
} 
Las ciencias biomédicas y las ciencias del comportamiento han demostrado en el último siglo un genuino esfuerzo por la investigación científica del estrés y sus mecanismos de acción sobre el organismo. Sin embargo, este esfuerzo empírico fue realizado de manera aislada y bajo los postulados cartesianos de la época, en los cuales los investigadores consideraban que las respuestas fisiológicas no tenían ningún correlato psicológico y viceversa, mostrando obviamente muy poco o ningún interés por el trabajo científico integrado y multidisciplinario. A comienzos del siglo XX, Walter Cannon desarrollaba sus estudios de fisiología en la Universidad de Harvard y describió por primera vez el fenómeno del estrés y el concepto de homeostasis (Cannon, 1915). Dos décadas más tarde, Hans Selye continuó los estudios de Cannon y se convirtió en la autoridad científica de la "respuesta fisiológica del estrés" (Selye, 1936). De manera paralela e independiente, Richard Lazarus inició sus estudios acerca del estrés en la Universidad de Berkeley, logrando significativos avances en su trabajo psicológico sobre el estrés y el afrontamiento (Lazarus, 1984).

A principios de la década de 1980 , estas dos disciplinas científicas comienzan, de manera estratégica y coordinada, un proceso de integración con el propósito de estudiar y entender adecuadamente los mecanismos psicobiológicos del fenómeno del estrés, en forma conjunta y multidisciplinaria. Este cambio sig- nificativo en la metodología de estudio e investigación del estrés responde al avance de la ciencia en tres importantes áreas. 1) El desarrollo de la neurociencia y el concepto de neuroplasticidad, el cual se refiere a los cambios sinápticos y de interacciones neuronales en el cerebro, debido a cambios comportamentales y psicosociales en el individuo. El concepto de neuroplasticidad reemplaza a la creencia antigua de que el cerebro es un órgano fisiológico "estático". 2) El avance en las técnicas de imágenes de resonancia magnética funcional (fMRI) y de tomografía por emisión de positrones (PET Scan), las cuales permiten estudiar las diferentes variables biológicas y sus correlatos comportamentales en el diseño de estudios de investigación científica. 3) La revolución genómica y epigenética, que viene demostrando la importancia de la interacción de los factores psicosociales y el ambiente en los mecanismos epigenéticos. El concepto central de la epigenética postula que la interacción de los genes y los factores ambientales determinan la salud del individuo (Lane \& Wager, 2009).

La programación de estudios multidisciplinarios que incluyen variables biomédicas y psicosociales ha estimulado el desarrollo del trabajo empírico y ha permitido un crecimiento vertiginoso del campo de la neurociencia y de la psicooncología. El concepto cartesiano de salud y enfermedad ha pasado a la historia, y con el inicio del siglo XXI, 
las ciencias biomédicas y las ciencias del comportamiento comparten nuevos modelos metodológicos y conceptuales multidisciplinarios para el continuo desarrollo de la investigación científica. Estos nuevos paradigmas permiten el estudio de las diferentes enfermedades relacionadas con el estrés, lo que genera un avance en el entendimiento de los mecanismos neurobiológicos de procesos metabólicos y mentales, incluyendo el cáncer.

El auge de esta labor multidisciplinaria ha posibilitado enormes beneficios. En primer lugar, las ciencias del comportamiento se fortalecen con base en el riguroso método científico tradicionalmente utilizado por las neurociencias, con el propósito de mejorar los métodos de investigación científica y los programas de intervención psicológica, como es el caso de la utilización de la terapia cognitiva centrada en mindfulness en la especialidad de neurociencias (Flook, Golberg, Pinger, Bonus \& Davidson, 2013) y de oncología (Moscoso, 2010). En segundo lugar, las ciencias del comportamiento contribuyen a la extensa producción empírica y de modelos conceptuales del estudio del estrés, afrontamiento y adaptación para el beneficio de los estudios biomédicos (Lazarus, 1993). En este sentido, dicho beneficio es mutuo, y ello se refleja en el gran avance científico observado actualmente en el campo de las neurociencias y de la psicología clínica de la salud.
El propósito de este artículo es presentar los fundamentos científicos de este nuevo modelo metodológico multidisciplinario para el estudio del estrés crónico, describiendo los mecanismos neurofisiológicos del distrés emocional percibido y su impacto en la salud del individuo. El segundo propósito es reportar las observaciones empíricas encontradas en relación con el proceso de adaptación de pacientes oncológicos en nuestros estudios de intervención mindfulness y el manejo del estrés crónico.

\section{LIMITACIONES METODOLÓGICAS DEL CONCEPTO DE ESTRÉS}

Una de las dificultades en el estudio del fenómeno del estrés está relacionada con la naturaleza efímera del concepto de estrés per se, el significativo número de connotaciones que ofrece, $\mathrm{y}$ la limitada utilidad para explicar cómo el organismo se adapta a situaciones adversas de la vida diaria. Es importante reiterar que el concepto de estrés no tiene una valoración definida y carece de especificidad, debido a su naturaleza fenomenológica y perceptiva. Mientras que para algunas personas el estrés representa un reto que motiva y estimula la obtención de logros y metas, para otras refleja un estado de preocupación, irritabilidad, temor y frustración para manejar adecuadamente las situaciones adversas de la vida diaria. Esta falta de especificidad 
del concepto de estrés no ha permitido una adecuada medición psicométrica, razón por la cual las ciencias del comportamiento sufren una notoria debilidad metodológica de investigación con respecto al estudio de este fenómeno.

El nuevo modelo multidisciplinario de investigación científica ha eliminado el estudio del estrés como fenómeno global, debido a esta debilidad metodológica y a la falta de precisión en la medición psicométrica. En la labor actual de investigación científica, es crítico reconocer que la claridad conceptual es esencial para el avance de la ciencia. Por lo tanto, el concepto de estrés crónico cumple un rol central dentro del nuevo modelo metodológico de investigación (Moscoso, 2009). En la actualidad, la gran mayoría de estudios en las áreas de neurociencias y psicología clínica de la salud utilizan modelos metodológicos que incluyen el estrés crónico como una noción fundamental en el contínuum de salud y enfermedad. Esta nueva visión en el proceso de investigación científica multidisciplinaria será descrita más ampliamente en líneas posteriores.

Una segunda limitación metodológica en el estudio del estrés global está relacionada con el antiguo concepto de homeostasis como noción central del proceso de adaptación. Este concepto fue inicialmente presentado por Walter Cannon (1915) y ampliamente descrito por Hans Selye (1936). La idea de adaptación homeostática fue el tema funda- mental en el modelo de estrés descrito por Cannon, en el cual se refiere a la estabilidad de los sistemas fisiológicos que mantienen la vida en estado de homeostasis (1932). Este sentido de estabilidad que Cannon le dio al concepto de homeostasis demostró ser un error metodológico en el estudio del estrés y adaptación. Contrario a esta noción de "estabilidad fisiológica", el avance de las ciencias biomédicas ha demostrado que la fisiología de los organismos se caracteriza por su dinamismo y cambio permanente dentro de un contínuum de parámetros especificos (McEwen \& Wingfield, 2003).

Este cambio reciente de dirección hacia un nuevo paradigma multidisciplinario de investigación de las ciencias biopsicosociales ha facilitado la inclusión de nuevas variables mediadoras neurobiológicas y psicosociales. Asimismo, presenta serias objeciones a la noción de homeostasis, por lo que plantea su reconsideración desde el punto de vista metodológico (Schulkin, 2003). En este sentido, las observaciones de las influencias del cerebro, el sistema nervioso central (SNC), y la inclusión de los principios de appraisal, aspectos cognitivos y estados emocionales como elementos fundamentales en el proceso de estrés, han permitido dejar de lado el concepto "estático" de homeostasis, estimulando el desarrollo de un nuevo marco teórico conocido como allostasis. 
Un NUEVO PARADIGMA DEL ESTRÉS:

ALLOSTASIS Y CARGA ALLOSTÁTICA

Sterling y Eyer (1988) presentan una exhaustiva revisión del principio de homeostasis descrito por Cannon y proponen un nuevo marco teórico para el estudio del estrés y la adaptación denominado allostasis. Allostasis se define como un proceso neurobiológico mediado directamente por el cerebro y el SNC con el propósito de mantener un nivel de estabilidad a través del cambio. Este proceso de allostasis facilita la adaptación del organismo a situaciones adversas o estresantes, debido a su naturaleza dinámica y multidisciplinaria, y a que permite un elevado grado de fluidez como respuesta a las diferentes demandas ambientales. Asimismo, posibilita un mejor entendimiento de las interacciones del cerebro, sistema nervioso, procesos cognitivos y emocionales como mediadores de la respuesta neurofisiológica del estrés (McEwen, 2006).

El concepto de allostasis es mucho más versátil que el concepto de homeostasis, por lo que es más útil para entender las interacciones neurobiológicas mediadas por el cerebro y el medio ambiente. En condiciones adversas y de estrés, los seres humanos y los animales, evolutivamente, cuentan con un complejo repertorio de respuestas fisiológicas y de comportamiento dirigidas a mantener una función protectora con el fin de sobrevivencia y adaptación (McEwen \& Wingfiel, 2003). El marco conceptual clásico del proceso homeostático no permite integrarse a la gran cantidad de evidencia empírica que indica que la respuesta neurofisiológica del estrés es fluida y de cambio constante, en función de la historia personal del individuo y su estilo de vida (Levine, 2001).

El concepto de allostasis permite una clara explicación del esfuerzo $\mathrm{acu}$ mulado de la respuesta neurofisiológica del estrés. Esta posibilidad facilita la introducción del principio de carga allostática como uno de los pilares de la teoría allostática (McEwen \& Stellar, 1993). McEwen y Stellar definen el concepto de carga allostática como el esfuerzo y desgaste físico causado por el exceso crónico de la actividad de los sistemas fisiológicos que cumplen un rol participatorio en el proceso de adaptación a situaciones de estrés. Este desgaste físico y mental cumple un rol central en el proceso de desarrollo de enfermedades físicas y mentales. Descrito en un lenguaje más simple: la carga allostática es el "precio que nuestro cuerpo y mente pagan por la presión constante de adaptarse a las situaciones adversas de la vida diaria causadas por el fenómeno de estrés crónico". Esta definición clara y concisa del concepto de carga allostática nos permite entender con mayor exactitud la importancia del estrés crónico y sus efectos acumulativos en la salud del individuo.

A pesar de que la carga allostática y el estrés crónico son dos conceptos 
independientes, ambos tienen una connotación similar en muchos aspectos del proceso de adaptación del organismo, particularmente en lo referido a los mecanismos neurofisiológicos que precipitan la pérdida de la salud. En ambos casos, el precio que el organismo paga por la presión constante y prolongada de estrés crónico se traduce en la alteración de neurotransmisores y hormonas de la respuesta neurofisiológica del estrés, como, por ejemplo, cortisol, adrenalina, insulina, vasopresina, endorfinas y corticotropina. Esta alteración se refleja en un aumento de la producción de algunas o en la insuficiencia de la liberación de otras, lo que causa, en última instancia, la pérdida de la salud del individuo (Koob, 1999). Un ejemplo clásico de este desbalance neurofisiológico fue reportado por Sapolski (1986), en el sentido de que los niveles elevados de cortisol causan una atrofia del hipocampo, lo cual genera alteraciones de la memoria y de ciertos procesos de aprendizaje.

\section{EL CONCEPTO DE ESTRÉS CRÓNICO}

El modelo científico del proceso de estrés reconoce la distinción entre el estrés agudo y el estrés crónico. Este último es el tema central del presente trabajo, particularmente porque tiene especial relevancia en el proceso de adaptación del individuo, en los rasgos de resiliencia y en los mecanismos neurofisiológicos relacionados con la salud y enfermedad. El estudio del estrés crónico ha recibido enorme atención en la última década debido a su elevada asociación con enfermedades crónicas, como, por ejemplo, la depresión, cefaleas, enfermedades metabólicas (obesidad/diabetes tipo 2), cáncer, enfermedades cardiovasculares, artritis reumatoide e hipertensión arterial, por mencionar algunas (Dallman, 2003).

El estrés crónico es causado por demandas y presiones durante periodos de tiempo prolongados, "percibidos" como eternos o interminables. En este sentido, la prevalencia del estrés crónico se observa notoriamente en grupos de personas expuestas a situaciones de pobreza, en personas obligadas al cuidado prolongado de ancianos o individuos con algún tipo de discapacidad, en personas que sufren de enfermedades crónicas, conflictos laborales, así como en aquellas que se encuentran en relaciones maritales disfuncionales. Algunos estudios empíricos en el área de neurociencia reportan niveles elevados de estrés crónico y alteraciones neurobiológicas en jóvenes y adultos con experiencias traumáticas en la etapa prenatal y desarrollo temprano. Estos estudios claramente nos indican que el estrés crónico no se inicia con el nacimiento de un individuo, sino muchas veces en la etapa de gestación (Lupien, McEwen, Gunnar \& Heim, 2009). Este tipo de experiencias adversas y estrés crónico en una edad temprana, por lo general, fomentan el desarrollo de una 
visión hostil y amenazante del mundo, facilitando la formación de un conjunto de actitudes negativas que determinan un estado de distrés emocional en el estilo de vida del individuo.

Desde el punto de vista de la neurociencia, se reconoce que la percepción individual o interpretación del estresor es de carácter eminentemente psicológico. Sin embargo, existe suficiente evidencia científica de que el estrés crónico tiene un correlato neurobiológico en el lóbulo frontal del cerebro. Asimismo, es necesario reconocer que la experiencia del distrés emocional percibi$d o$ se inicia en el cerebro como órgano mediador de la respuesta neurofisiológica del estrés. La experiencia de distrés emocional causada por el estrés crónico es un proceso cognitivo que incluye un desbalance en la formación de emociones negativas y positivas, inclinando la balanza hacia un incremento de emociones negativas y el consecuente desencadenamiento de cuadros de depresión clínica. Esta desregulación emocionalcognitiva del individuo cumple un rol desencadenante en una serie de mecanismos de activación neurobiológica del sistema límbico, y de manera particular en el lóbulo prefrontal, amígdala e hipocampo (McEwen, 2007).

El estudio científico del estrés crónico requiere de una claridad conceptual con el propósito de medir esta variable psicométricamente. Es así que las ciencias del comportamiento necesitan dis- tinguir operacional y empíricamente el concepto de estrés crónico y los factores o características directamente relacionadas con este constructo, es decir, los síntomas de distrés emocional que forman parte del concepto de "estresado" (Moscoso \& Reheiser, 2010). La construcción y desarrollo del Inventario de Distrés Emocional Percibido (IDEP) es el resultado de este avance científico y de la necesidad de contar con una medida psicométrica válida y confiable para la evaluación del distrés emocional como un correlato directo del estrés crónico (Moscoso, 2011).

\section{Un PARÁMETRO CLÍNICO del ESTRÉS CRÓNICO: DISTRÉS EMOCIONAL}

El distrés emocional percibido es conceptualizado como un cuadro clínico asociado al estrés crónico, que constituye un tema de investigación en neurociencias. Conceptualmente, el distrés emocional se define como "un estado marcado por sentimientos que varían en intensidad, desde tristeza, inseguridad, confusión y preocupación, hasta la experiencia de síntomas mucho más severos como, por ejemplo, la ansiedad, depresión, ira, aislamiento social y pérdida de esperanza" (Moscoso, McCreary, Goldenfarb, Knapp \& Reheiser, 2000).

Nuestra línea de investigación psicométrica en pacientes con cáncer muestra que el concepto de distrés emocional percibido incluye ansiedad, ira, pérdida de esperanza y un conjunto de síntomas 
depresivos que deben ser claramente distinguidos del diagnóstico de una depresión severa (Moscoso, Lengacher \& Reheiser, 2012). Estudios realizados por Cassileth, Lusk, Hutter, Strouse \& Brown (1984) sugieren que los síntomas de ansiedad y depresión representan simplemente "dos rótulos aparentemente diferentes" con un elevado nivel de correlación. Ello nos indica que la ansiedad y la depresión clínica "marchan de la mano", lo cual está claramente demostrado en la práctica clínica.
El IDEP, en su forma actual, es el resultado de esfuerzos de investigación psicométrica en la Universidad del Sur de Florida durante la última década. Nuestro propósito fue medir el distrés emocional como un correlato clínico del estrés crónico y ofrecer un instrumento válido en el área de neurociencias y psicología clínica de la salud. Este instrumento tiene la propiedad de evaluar las diferentes dimensiones de la ansiedad, depresión, expresión de la ira y desesperanza en personas que están en condi-

Figura 1. Modelo conceptual y estructura factorial del Inventario de Distrés Emocional Percibido

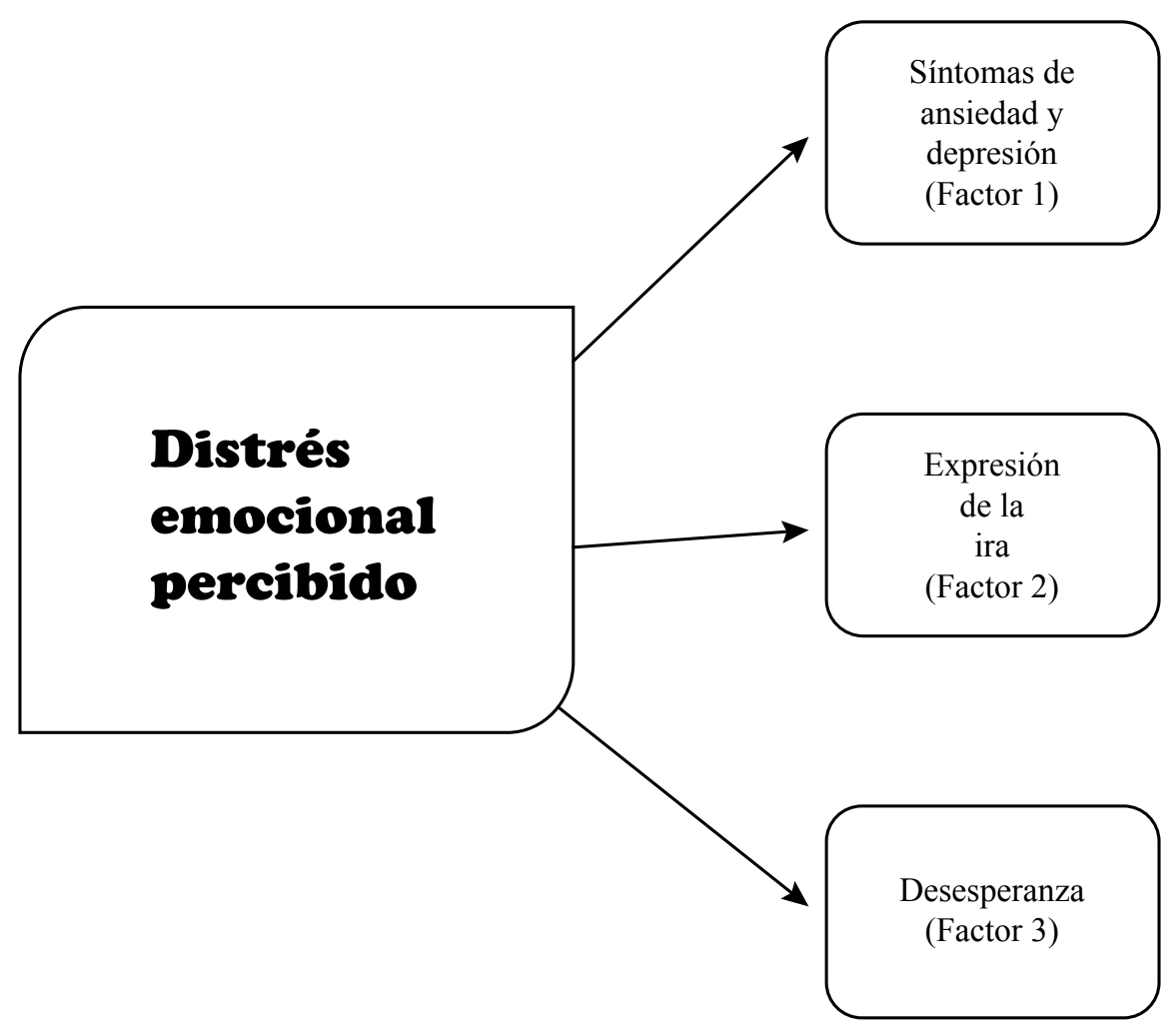


ciones de estrés crónico, particularmente, pacientes con diagnóstico de cáncer. Esta prueba psicométrica es sensible a la naturaleza multidimensional del concepto de distrés emocional y considera la medición de un índice global de distrés emocional percibido con base en la multidimensionalidad de este concepto.

\section{EFECTOS NEUROBIOLÓGICOS DEL ESTRÉS CRÓNICO EN LA SALUD}

El cerebro tiene un enorme control sobre el SNC en la regulación de neurotransmisores y hormonas; sobre el sistema endocrino, en la regulación de corticoesteroides; sobre el sistema inmune, en la regulación de citoquinas proinflamatorias, y en los cromosomas, en la regulación de telómeros y telomerasa. Debido a los efectos acumulativos del estrés crónico en la vida diaria de una persona, los mediadores bioquímicos estimulados por la respuesta neurofisiológica del estrés cumplen un rol opuesto a su función inicialmente protectora, generando una desregulación neurobiológica que altera el equilibrio allostático y produce una carga allostática. Por ejemplo, el exceso de cortisol inhibe la producción de insulina en el páncreas, lo que explica claramente las interacciones del estrés crónico, los niveles de cortisol y la resistencia insulínica en los casos de la diabetes tipo 2. Igualmente, el exceso de cortisol produce una crisis energética en las neuronas del cerebro debido a que el almacenamiento de glucosa en las célu- las de grasa inhibe el $75 \%$ de absorción de glucosa en el cerebro, y esto genera dicha crisis energética en el individuo. Este proceso explica la interacción neurobiológica del estrés crónico, los niveles elevados de cortisol y la obesidad.

El cerebro ejerce también un control sobre las emociones y la adaptación del individuo en el proceso de estrés crónico. El distrés emocional experimentado en situaciones de adversidad tiene un correlato neurobiológico en el lóbulo prefrontal del cerebro, lo cual nos permite entender que la experiencia del estrés se inicia y afecta al cerebro de manera directa (McEwen, 1998; Sapolsky, 1996). La percepción cognitiva de la persona, formada en el lóbulo frontal del cerebro, determina ciertamente lo que es "estresante".

Cuando la experiencia de adversidad se convierte en un proceso de estrés crónico, esta facilita el inicio de un estado de distrés emocional, en el que se observa una excesiva utilización $\mathrm{o}$, a veces, un manejo ineficiente de ciertas hormonas, catecolaminas y corticoesteroides, por parte del eje hipotalámico-pituitariosuprarrenal (HPA Axis) y el eje simpático-suprarrenal-medular (SAM Axis). Dicho estado de distrés emocional, causado por la experiencia de estrés crónico, produce un impacto negativo en el SNC, activando cambios neurofisiológicos y un desbalance hormonal que afecta a los sistemas endocrino e inmune (Dhabhar \& McEwen, 1997). Esta 
cascada neurofisiológica nos permite observar la naturaleza multidireccional de las interacciones entre la percepción de un evento estresante, el cerebro, y los sistemas endocrino e inmune (Sapolski, Romero \& Munck, 2000).

Estas alteraciones neurobiológicas causadas por el estrés crónico en los sistemas reguladores de glucocorticoides, catecolaminas y hormonas del SNC provocan un desgaste en las múltiples funciones cerebrales, endocrinas e inmunológicas, precipitando un gran número de enfermedades físicas y mentales. Un significativo número de estudios empíricos han demostrado que el estrés crónico produce una pérdida de neuronas en el hipocampo, lo cual resulta en una reducción significativa del volumen de esta estructura cerebral. Está demostrado que dicha alteración estructural del hipocampo ocasiona pérdida de la memoria y, por consiguiente, afecta los procesos de aprendizaje. Asimismo, existe evidencia científica de que el estrés crónico genera, opuestamente, un alargamiento de la amígdala que causa niveles elevados de ansiedad y cuadros obsesivo-compulsivos (McEwen, 2001). Estos mismos estudios reportan una supresión en las defensas del sistema inmune y síntomas relacionados con la fatiga de las glándulas suprarrenales, provocados específicamente por la reducción de cortisol. Es importante precisar que el estrés crónico tiene una influencia directa en los procesos inflamatorios del organismo, pues produce una elevación de citoquinas proinflamatorias, lo cual provoca alergias respiratorias, asma, artritis reumatoide, depresión e insomnio (MacPherson, Dinkel \& Sapolski, 2005).

Desde el punto de vista metabólico, el estrés crónico induce al consumo excesivo de calorías; esto provoca el incremento de cortisol y el almacenamiento de glucosa en las células grasas, lo cual favorece el desarrollo de la obesidad y la diabetes tipo 2 (Epel, Lapidu \& McEwen, 2000). En este mismo sentido, la experiencia del estrés crónico facilita la adquisición de estilos de vida poco saludables, que contribuyen a empeorar la situación de distrés emocional de la persona, de manera que se genera una especie de círculo vicioso. Estilos de vida que fomentan el uso excesivo de alcohol, el consumo de tabaco y drogas, y un consumo elevado de calorías y grasas saturadas (Armeli, Todd \& Mohr, 2005).

La riqueza empírica de las ciencias del comportamiento en el estudio del estrés crónico ha contribuido a un mejor entendimiento de los conceptos de ambiente social y estilos de vida como factores mediadores y moduladores del impacto negativo de este fenómeno en la salud física y mental del individuo. Está científicamente establecido que el impacto negativo del estrés crónico propicia el desarrollo de un significativo número de enfermedades de carácter sistémico, mejor conocidas como las enfermedades del siglo XXI: obesidad, depresión, fibromialgia, artritis reumatoide, fatiga crónica, enfermedades cardiovasculares, 
hipertensión, diabetes tipo 2, síndrome metabólico, asma y cáncer, solo por incluir las más comunes.

\section{REDUCCIÓN DEL ESTRÉS CRÓNICO: DEL} LABORATORIO A LA CABECERA DEL PACIENTE

La investigación científica traslacional fue desarrollada con la intención de unir lazos entre los estudios de investigación básica y la labor empírica de aplicación clínica (from the bench to the bedside). La adquisición de nuevos conocimientos científicos acerca de los mecanismos neurofisiológicos de la respuesta del estrés crónico se ha traducido en la implementación de la terapia cognitiva centrada en mindfulness como una de las formas de tratamiento en neurociencias y en psicología clínica de la salud. Este programa de intervención psicológica enfatiza la prevención del distrés emocional dentro del proceso de adaptación al estrés crónico, tomando en consideración los factores neurobiológicos y psicológicos que cumplen un rol importante en este proceso. Este tipo de intervención psicológica ofrece entrenamiento en la reducción del estrés crónico, la respuesta de relajación y la modificación de los patrones cognitivos autodestructivos, facilitando el desarrollo de emociones positivas (Garland, Gaylord \& Fredrickson, 2011). Asimismo, permite el desarrollo de un estilo de vida saludable con la intención de promover la calidad de vida del paciente (Moscoso, Reheiser \& Hann, 2004).

Figura 2. Marco conceptual de la terapia cognitiva centrada en Mindfulness

\section{Mindfulness y distrés emocional}

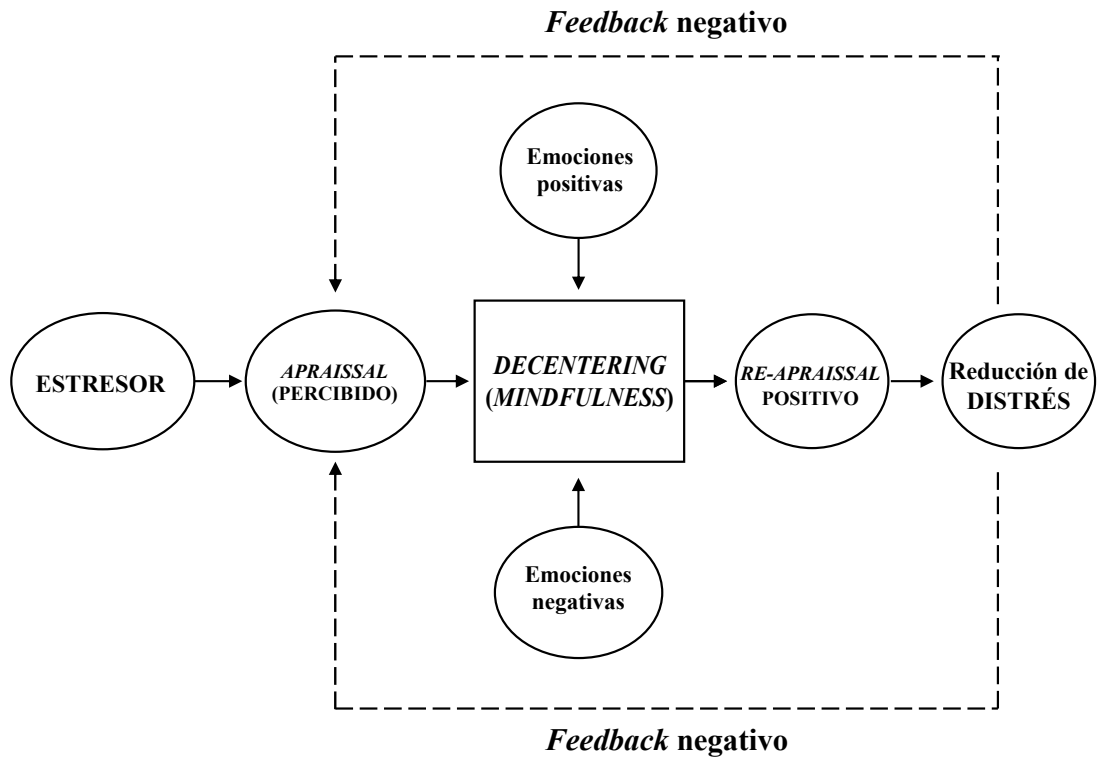


El manejo psicobiológico del estrés crónico con base en la práctica de mindfulness estimula el aprendizaje de la respuesta de relajación como una técnica mente-cuerpo. Igualmente, este programa terapéutico ayuda al manejo cognitivo de la depresión clínica y evita la posible recurrencia de esta enfermedad (Segal, Williams \& Teasdale, 2001). Bishop (2002) reporta que los pacientes entrenados en mindfulness logran manejar adecuadamente sus niveles de distrés emocional y sus reacciones somáticas frente a situaciones de estrés crónico. Speca et al. (2000) reportaron que mindfulness es efectivo en reducir síntomas de distrés emocional, en comparación con el grupo de control. Los estudios dirigidos por Carlson et al. (2007) muestran que el programa de reducción de estrés basado en mindfulness mejora la calidad de vida de pacientes con cáncer y reduce los niveles de distrés emocional. Estos estudios, además, reportaron una regulación óptima de los patrones de cortisol en los pacientes asignados al grupo de tratamiento, en comparación con los pacientes asignados a los grupos de control. Un estudio dirigido por Moscoso, Reheiser \& Hann (2004) reportó una reducción significativa en los niveles de ansiedad, depresión y distrés emocional en un grupo de pacientes con cáncer de mama.

Los estudios científicos mencionados en el párrafo anterior estimularon nuestro interés por seguir evaluando la eficacia de la terapia de reducción del estrés crónico sobre la base del programa de mindfulness. Con el apoyo financiero del Instituto Nacional de Salud de los Estados Unidos, iniciamos una serie de investigaciones clínicas en la Universidad del Sur de Florida con el propósito de continuar evaluando los beneficios de este programa terapéutico. Las metas propuestas en nuestros estudios tienen como objetivo observar los cambios cognitivos y neurobiológicos en pacientes oncológicos. La metodología de investigación utilizada en nuestros estudios responde a los criterios de integración multidisciplinaria descritos a inicios del presente artículo, y plantea la posibilidad de observar las diferencias clínicas en 1) variables cognitivas, como ansiedad, depresión, temor, distrés; en 2) variables somáticas, como, por ejemplo, fatiga, dolor, insomnio; y en 3) variables neurobiológicas, como es el caso del cortisol, las citoquinas proinflamatorias y la telomerasa.

En relación con las variables cognitivas, nuestro primer reporte publicado en el Journal of Psycho-Oncology observó una significativa reducción de los síntomas de distrés emocional y el temor de recurrencia del tumor maligno en pacientes con cáncer de mama. De igual manera, se notó que los pacientes asignados al grupo de tratamiento presentaron un aumento en los niveles de energía y funcionamiento físico, en comparación con el grupo de control (Lengacher et al., 2009). 
En cuanto al estudio de las variables somáticas, un reporte empírico de nuestro equipo de trabajo publicado en el Journal of Behavioral Medicine indica que el tratamiento mindfulness reduce síntomas gastrointestinales y fatiga crónica en pacientes oncológicos, es decir, un significativo mejoramiento de la calidad de vida del paciente (Lengacher et al., 2012).

El estudio de las variables neurobiológicas y las hormonas es parte integral de nuestra investigación. Es así que un estudio piloto realizado en pacientes con diagnóstico avanzado de cáncer y un familiar responsable de su cuidado nos permitió observar que la terapia mindfulness es beneficiosa. Los pacientes participantes del tratamiento reportaron mejoría en términos del nivel de estrés percibido. Los exámenes de cortisol y citoquinas proinflamatorias (IL-6) tomados antes y después del tratamiento mindfulness indicaron una reducción de ambos componentes neurofisiológicos en los pacientes y los familiares participantes del estudio (Lengacher et al., 2012).

En una reciente publicación en el Journal of Biological Research for Nursing, presentamos un reporte en el cual se indica que el tratamiento mindfulness en un grupo de pacientes con cáncer de mama logra afectar positivamente la recuperación de células $\mathrm{T}$ en el sistema inmune, así como un mejoramiento de los promedios de células inmunológicas
Th1/Th2, después de haber culminado los tratamientos de quimioterapia y radiación (Lengacher et al., 2013).

Un último reporte de variables neurobiológicas, publicado en el Journal of Biological Research for Nursing en el 2014, muestra nuestras observaciones respecto a que el estudio de randomización controlada en pacientes con cáncer de mama ofrece evidencia empírica de que la terapia mindfulness eleva los niveles de telomerasa (una enzima cromosómica) en este tipo de pacientes. Este mismo reporte indica que este aumento de telomerasa es evidente desde un comienzo del tratamiento mindfulness hasta su última evaluación en la semana 12 (week 12). Se concluye que la recuperación del estado psicológico y emocional de los pacientes está asociada, en gran medida, con este aumento de telomerasa (Lengacher et al., 2014).

\section{SUMARIO Y CONCLUSIONES}

El estudio científico del estrés ha iniciado una vigorosa etapa en su desarrollo gracias al esfuerzo multidisciplinario de las ciencias biomédicas y del comportamiento. El auge de esta labor integradora ha permitido que las ciencias del comportamiento se beneficien del riguroso método científico tradicionalmente utilizado por las neurociencias, y, a su vez, las ciencias biomédicas aprovechan la extensa producción empírica y el marco conceptual del proceso de estrés, afrontamiento y 
adaptación desarrollado por las ciencias del comportamiento. En este sentido, el beneficio es mutuo, y ello refleja el gran avance científico observado actualmente en el campo de las neurociencias y de la psicología clínica de la salud.

Este nuevo modelo multidisciplinario de investigación científica ha dejado de lado el concepto de estrés en su forma abstracta, debido a su debilidad metodológica y la falta de precisión en medición psicométrica. En la actualidad, se considera al estrés crónico como un nuevo postulado metodológico de investigación. Desde este nuevo ángulo multidisciplinario, las observaciones de las influencias del cerebro y el SNC, incluyendo los principios de appraisal, en los aspectos cognitivos y estados emocionales han dejado de lado el concepto "estático" de homeostasis, para permitir el advenimiento de un nuevo marco conceptual denominado allostasis y carga allostática.

El estrés crónico ha recibido una gran consideración en el campo de la investigación científica debido a su elevada correlación con enfermedades crónicas, como es el caso de la depresión, cefaleas, obesidad, diabetes tipo 2, enfermedades cardiovasculares, hipertensión arterial, artritis y cáncer. Las alteraciones neurobiológicas causadas por el estrés crónico en los sistemas reguladores de glucocorticoides, catecolaminas y hormonas generan un desgaste en las múltiples funciones cerebrales, endocrinas e inmunológicas, precipitando dichas enfer- medades físicas y mentales. El distrés emocional, como resultado de la experiencia de estrés crónico en el individuo, es causado por un desbalance cognitivo de emociones negativas y positivas.

El avance científico en el entendimiento de los mecanismos neurobiológicos de la respuesta del estrés crónico ha facilitado la implementación de la terapia cognitiva centrada en mindfulness como un programa de tratamiento en neurociencias y en psicología clínica de la salud. Las aplicaciones clínicas de este programa terapéutico son efectivas en el contexto del estrés crónico, pues alivian la depresión y reducen el distrés emocional con el propósito de crear un nuevo estilo de vida y mejorar la salud del individuo (Lengacher et. al., 2007).

Este tipo de intervención psicológica enfatiza la prevención del distrés emocional dentro del proceso de adaptación al estrés crónico, y toma en consideración los factores neurobiológicos y psicológicos que cumplen un rol central en el proceso de gestación del estrés crónico. La meta de la terapia mindfulness es asistir a la persona en la reestructuración de situaciones adversas y estresantes, creando una percepción positiva de oportunidad, crecimiento personal y mejora en la calidad de vida. Esta forma de entrenamiento cognitivo, denominada decentering, posibilita un cambio de interpretación de los eventos estresantes con el propósito reducir el nivel de distrés emocional, y elevar los niveles de optimismo y autocompasión. 


\section{REFERENCIAS}

Armeli, S., Todd, M., Mohr, C. (2005). A daily process approach to individual differences in stress-related alcohol use. Journal of Personality, 73, 6, 1-30.

Bishop, S. R. (2002). What do we really know about mindfulness-based stress reduction? Psychosomatic Medicine, 64, 71-83.

Cannon, W. B. (1915). Bodily Changes in Pain, Hunger, Fear and Rage. Boston, MA: C.T. Branford Co., Inc.

Cannon, W. B. (1932). The Wisdom of the Body. Nueva York: W.W. Norton and Co., Inc.

Carlson, L. E., Speca, M., Patel, K. D., Faris, P. (2007). One year pre-post intervention follow-up of psychological, immune, endocrine and blood pressure outcomes of mindfulnessbased stress reduction (MBSR) in breast and prostate cancer outpatients. Brain, Behavior and Immunology, 21, 1038-1049.

Cassileth, B. R., Lusk, E. J., Hutter, R., Strouse, T. B., \& Brown, L. I. (1984). Concordance of depression and anxiety in patients with cancer. Psychological Reports, 54, 588-590.

Dallman, M. F. (2003). Chronic stress and obesity: A new view of "comfort food". Proccedings of the $\mathrm{Na}$ tional Academy of Science, 100, 11696-11701.
Dhabhar, F. S., McEwen, B. S. (1997). Acute stress enhances while chronic stress suppresses cell-mediated immunity in vivo: A potential role for leukocyte trafficking. Brain, Behavior and Immunity, 114, 286-306.

Epel, E., Lapidus, R., McEwen, B. (2000). Stress may add bite to appetite in women: A laboratory study of stress-induced cortisol and eating behavior. Psychoneuroendocrinology, 26, 37-49.

Flook, L., Goldberg, S. B., Pinger, L., Bonus, K., \& Davidson, R. J. (2013). Mindfulness for teachers: A pilot study to assess effects on stress, burnout and teaching efficacy. Mind, Brain and Education, 7, 182-195. Doi: 10.1111/mbe.12026. PMCID: PMC3855679.

Garland, E. L., Gaylord, S. A., \& Fredrickson, B. L. (2011). Positive appraisal mediates the stressreductive effects of mindfulness: An upward spiral process. Mindfulness, 2, 59-67.

Koob, G. F. (1999). Corticotropinreleasing factor, norepinephrine and stress. Biological Psychiatry, 46, 1167-1180.

Lane, R. D., \& Wager T. D. (2009). The new field of brain-body medicine: What have we learned and where are we headed? NeuroImage, 47, 11351140. 
Lazarus, R. S. (1993). Coping theory and research: Past, present and future. Psychosomatic Medicine, 55, 3, 234-247.

Lazarus, R. S., \& Folkman, S. (1984). Stress, Appraisal and Coping. Nueva York: Springer.

Lengacher, C. A., Kip, K. E., Moscoso, M., Johnson-Mallard, V., Molinari, M., Gaurkee, D., \& Greenberg, H. (2007). Mindfulness-Based Stress Reduction (MBSR) improves psychological status and general health among breast cancer survivors. Psycho-Oncology, 16, 3, S72-S73.

Lengacher, C. A., Kip, K., Barta, M., Jacobsen, P. B., Groer, M., Lehman, B., \& Molinary, M. S. (2012). A pilot study evaluating the effects of Mindfulness Based Stress Reduction on psychological status, physical status, salivary cortisol, Interleukin-6 among advanced-stage cancer patients and their caregivers. Journal of Holistic Nursing, 30, 170-185.

Lengacher, C. A., Kip, K., Post-White, J., Fitzgerald, S., Newton, C., Barta., \& Jacobson, P. B. (2013). Lymphocyte Recovery after Breast Cancer Treatment and Mindfulness-Based Stress Reduction (MBSR) Therapy. Journal of Biological Research for Nursing, $15,1,37-47$.

Lengacher, C., Johnson-Mallard, V., PostWhite, J., Moscoso, M., Jacobsen, P., Klein, T., \& Kip, K. (2009). Random- ized controlled trial of mindfulnessbased stress reduction (MBSR) for survivors of breast cancer. PsychoOncology, 18, 12, 1261-1272. Doi: 10.1002/pon.1529: 10.1002/pon.1529.

Lengacher, C. A., Reich, R. R., Kip, K. E., Barta, M., Ramesar, S., Paterson, C. L., \& Park, J. Y. (2014). Influence of Mindfulness-Based Stress Reduction on Telomerase Activity in Women with Breast Cancer (BC). Biological Reseach for Nursing. Doi: 10. 1177.

Lengacher, C., Reich, R., Post-White, J., Moscoso, M., Shelton, M., Barta, M., \& Budhrani, P. (2012). Mindfulness based stress reduction in posttreatment breast cancer patients: An examination of symptoms and symptom clusters. Journal of Behavioral Medicine, 35, 86-94. Doi: 2011.10.1007/s10865-011-9346-4: 10.1007/s10865-011-9346-4.

Levine, S. (2001). Primary social relationships influence the development of the hypothalamic-pituitary-adrenal axis in the rat. Physiology \& Behavior, 73, 255-260.

Lupien, S. J., McEwen, B. S., Gunnar, M. R., \& Heim, C. (2009). Effects of the stress throughout the lifespan on the brain, behavior and cognition. Nature Reviews Neuroscience, 10, 6, 434-445.

MacPherson, A., Dinkel, K., \& Sapolsky, R. (2005). Glucorticoids worsen ex- 
citotoxin-induced expression of proinflammatory cytokines in hippocampal cultures. Experimental Neurology, 194, 376-383.

McEwen, B. S. (1998). Protective and Damaging Effects of Stress Mediators. New England Journal of Medicine, 338, 171-179.

McEwen, B. S. (2001). Plasticity of the hippocampus: adaptation to chronic stress and allostatic load. Annals of the New York Academy of Sciences, 933, 265-277.

McEwen, B. S. (2006). Sleep deprivation as a neurobiologic and physiologic stressor, allostastis and allostatic load. Metabolism, 55, S20-S23.

McEwen, B. S. (2007). The physiology and neurobiology of stress and adaptation: Central role of the brain. Physiological Reviews, 87, 873-904.

McEwen, B. S., \& Stellar, E. (1993). Stress and the individual: Mechanisms leading to disease. Archives of Internal Medicine, 153, 2093-2101.

McEwen, B. S., \& Wingfield, J. C. (2003). The concept of allostasis in biology and biomedicine. Hormones \& Behavior, 43, 2-15.

Moscoso, M. S. (2009). De la mente a la célula: impacto del estrés en psiconeuroinmunoendocrinología. Revista Liberabit, 15, 2, 143-152.

Moscoso, M. S. (2010). El estrés crónico y la terapia cognitiva centrada en mindfulness: una nueva dimensión en psiconeuroinmunología. Revista Persona, 13, 11-29.

Moscoso, M. S. (2011). El estrés crónico y la medición psicométrica de distrés emocional percibido en medicina y psicología clínica de la salud. Revista Liberabit, 17, 1, 67-76.

Moscoso, M. S., Lengacher, C. A., \& Reheiser, E. C. (2012). The assessment of the perceived emotional distress: The neglected side of cancer care. Journal of Psicooncologia, 9, 2-3, 277-288.

Moscoso, M. S., McCreary, D., Goldenfarb, P., Knapp, M., \& Reheiser, E. (2000). A brief screening inventory to measure emotional distress in cancer patients. Psycho-Oncology, 9, 5, S53.

Moscoso, M. S., \& Reheiser, E. C. (2010). Construct validity of the Emotional Distress Inventory in patients with cancer. Ansiedad y Estrés, 16, 1, 83-94.

Moscoso, M. S., Reheiser, E. C., \& Hann, D. (2004). Effects of a brief mindfulness-based stress reduction intervention on cancer patients. Psycho-Oncology, 13, 1, S12.

Sapolsky, R. M. (1986). Glucocorticoid toxicity in the hippocampus reversal by supplementation with brain fuels. The Journal of Neuroscience, 6, 2240-2244.

Sapolsky, R. M. (1996). Why stress is bad for your brain. Science, 273, 749-750. 
Sapolsky, R. M., Romero, L. M., \& Munck, A. U. (2000). How do glucocorticoids influence stress responses? Integrating permissive, suppressive, stimulatory and preparative actions. Endocrine Review, 21, 55-89.

Schulkin, J. (2003). Rethinking homeostasis: Allostatic regulation in physiology and pathophysiology. Cambridge, MA: MIT Press.

Segal, Z. V., Williams, M. G., \& Teasdale, J. D. (2001). Mindfulness-Based Cognitive Therapy for Depression: A New Approach to Preventing Relapse. Columbus, $\mathrm{OH}$ : The Guilford Press.
Selye, H. (1936). A syndrome produced by diverse nocuous agents. Nature, 138, 32 .

Speca, M., Carlson, L. E., Goodey, E., \& Angen, M. (2000). A randomized, wait-list controlled clinical trial: The effect of a mindfulness meditationbased stress reduction program on mood and symptoms of stress in cancer outpatients. Psychosomatic Medicine, 62, 613-622.

Sterling, P., \& Eyer, J. (1988). Allostasis, a new paradigm to explain arousal pathology. En S. Fisher, \& J. Reason (Eds.), Handbook of life stress, cognition and health (pp. 629-649). Nueva York: John Wiley \& Sons. 